\title{
Online NARMAX model for electron fluxes at GEO
}

\author{
R. J. Boynton, M. A. Balikhin, and S. A. Billings \\ Department of Automatic Control and Systems Engineering, University of Sheffield, Sheffield S1 3JD, UK
}

Correspondence to: R. J. Boynton (r.boynton@ sheffield.ac.uk)

Received: 14 August 2014 - Revised: 13 January 2015 - Accepted: 6 March 2015 - Published: 27 March 2015

\begin{abstract}
Multi-input single-output (MISO) nonlinear autoregressive moving average with exogenous inputs (NARMAX) models have been derived to forecast the > $0.8 \mathrm{MeV}$ and $>2 \mathrm{MeV}$ electron fluxes at geostationary Earth orbit (GEO). The NARMAX algorithm is able to identify mathematical model for a wide class of nonlinear systems from input-output data. The models employ solar wind parameters as inputs to provide an estimate of the average electron flux for the following day, i.e. the 1-day forecast. The identified models are shown to provide a reliable forecast for both $>0.8$ and $>2 \mathrm{MeV}$ electron fluxes and are capable of providing real-time warnings of when the electron fluxes will be dangerously high for satellite systems. These models, named $\mathrm{SNB}^{3} \mathrm{GEO}>0.8$ and $>2 \mathrm{MeV}$ electron flux models, have been implemented online at http://www.ssg.group.shef. ac.uk/USSW/UOSSW.html.
\end{abstract}

Keywords. Magnetospheric physics (energetic particles trapped; solar wind-magnetosphere interactions)

\section{Introduction}

The configuration of the magnetic field in the region of the terrestrial radiation belts allows for charged particles to be trapped. As such, the radiation belts contain energetic electron from tens of $\mathrm{keV}$ to several $\mathrm{MeV}$. The population of the energetic electrons can vary by large amounts in very short timescales (Blake et al., 1992; Reeves, 1998). High fluxes of the energetic electrons can cause problems for modern technological systems and can be hazards for humans in space. Satellites in both low Earth orbit and geostationary Earth orbit (GEO) have an increased probability of suffering onboard satellite system malfunctioning, which can result in permanent hardware damage (Reagan et al., 1983; Baker et al., 1987). By powering down certain systems that are at risk, the effects of the energetic particles can be mitigated. However, this will require prior knowledge of high energetic electron populations that are dangerous to satellites. Therefore, models are needed to forecast when large fluxes of highly energetic electrons will occur.

Although the radiation belts were discovered more than half a century ago (Van Allen, 1959), during the very first in situ space measurements, there still lacks a comprehensive physical model of the solar wind interaction with the terrestrial magnetosphere and the dynamics of the radiation belts. Reeves (1998) investigated the relationship between the disturbances within the magnetosphere and radiation belts, which was found to be very complex. About half of the magnetic storms led to a significant increase in electron fluxes, a quarter resulted in a decrease while the final quarter of the disturbances had no effect on the electron fluxes. A similar study, performed by Kataoka and Miyoshi (2006), showed that while a southward interplanetary magnetic field (IMF) gave rise to $49 \mathrm{CME}$ magnetospheric disturbances, only 21 of these resulted in increases of energetic electrons. Although, in stream interaction regions associated with disturbances, they recorded an $83 \%$ probability of an electron flux increase.

Despite the complexity of the particle acceleration within the radiation belts, there are a number of proposed models that explain the dynamics (Friedel et al., 2002; O'Brien et al., 2003). Some models assume that a combination of radial diffusion and low-altitude ULF waves lead to a recirculation effect, repeatedly accelerating the particles (Fujimoto and Nishida, 1990; Liu et al., 1999; Boscher et al., 2000). The diffusion of trapped energetic electrons from the cusp into radiation belts has been suggested by Sheldon et al. (1998). Temerin et al. (1994), Li et al. (1997) and Summers et al. (1998) all advocate electron cyclotron heating by whistler waves. An enhanced earthward transport from the tail to GEO by repetitive substorm injections was put forward by Ingraham et al. (2001). Baker et al. (1979) proposed the more 
exotic explanation of the Jovian shock increasing the electron population in the Earth's radiation belts. The review by Friedel et al. (2002) includes all these mechanisms for acceleration. The main candidates are based on either local diffusion due to particle interactions with waves (Temerin et al., 1994; Shprits et al., 2008; Reeves et al., 2009; Omura et al., 2007; Horne et al., 2005; Summers and Thorne, 2003; Summers et al., 1998, 2002, 2004; Albert, 2003, 2005) or radial diffusion (Falthammar, 1968; Schulz and Lanzerotti, 1974; Hilmer et al., 2000; Ingraham et al., 2001; McAdams et al., 2001).

An alternative approach to developing a model based on first principles is to deduce a forecasting model for the radiation belt electron fluxes directly from data (Baker et al., 1990; Freeman et al., 1998; Wei et al., 2011). Baker et al. (1990) employed linear prediction filters (LPFs) to characterize and to predict the $3-40 \mathrm{MeV}$ electron measurements using solar wind velocity and geomagnetic indices such as $\mathrm{Kp}$ and $\mathrm{AE}$ as inputs. The use of LPFs allowed the authors to analyse how the inputs influence the output, reporting a 27 day periodicity and a peak lag at 2-3 days. Since this study, most of the data analysis research into energetic electrons has been accomplished by neural networks (NNs). The inputs to the NNs are often the geomagnetic indices such as the Dst index and the daily sum of the global geomagnetic index, Kp (Freeman et al., 1998; Ling et al., 2010; Koons and Gorney, 1991). NNs have provided results that are significantly more accurate than those from LPFs; however, the NNs are much more difficult to interpret than the LPFs. They result in a complex array of neurons, each with an activation function, linked through a maze of other neurons by a set of weights. This makes the relationship between the input and output very hard to understand.

Nonlinear autoregressive moving average with exogenous inputs (NARMAX) models have the advantage of providing accurate results and at the same time are very easy to interpret. The NARMAX algorithm was initially developed for complex engineering and biological systems but has since been employed in many other fields, such as space weather. In solar-terrestrial physics, the NARMAX methodology was first employed to develop forecasting models for the Dst index using solar wind inputs (Balikhin et al., 2001; Boaghe et al., 2001). NARMAX has also been used to model the electron fluxes at GEO (Wei et al., 2011). Recently, the NARMAX approach has been used to identify the inputs of a natural dynamical system for cases when there is an absence of knowledge. The error reduction ratio (ERR), which is the basis of the NARMAX model structure selection, was used by Boynton et al. (2011b) to analyse how the previously proposed coupling functions influence the Dst index. This study produced a new coupling function that was then used as an input to model the Dst index in a following paper (Boynton et al., 2011a). This technique has recently been applied to various energies of the electron flux ranging from $24.1 \mathrm{keV}$ to $3.5 \mathrm{MeV}$, obtaining some unexpected results (Boynton et al.,
2013; Balikhin et al., 2011). Balikhin et al. (2011) found that for $1.8-3.5 \mathrm{MeV}$ electrons, the solar wind density had the most influence. The following study by Boynton et al. (2013) reported an increasing influence in density from $\sim 1$ until $1.8 \mathrm{MeV}$, above which it became the most important control parameter for the electron fluxes at GEO. They also identified a quantitative timescale of the electron flux enhancement as a function of energy that allowed Balikhin et al. (2012) to argue that local diffusion is not dominant at GEO. Since there are time delays between the solar wind velocity increases and the electron flux enhancements at GEO, it is possible to quantitatively estimate the 1-day-ahead electron fluxes from solar wind parameters. The work by Boynton et al. (2013) indicates that this is possible for energies above $270 \mathrm{keV}$, where they report that the velocity of the previous day is the parameter that has the most influence on the fluxes.

The main aim of this study was to develop two NARMAX models for the $>0.8$ and $>2 \mathrm{MeV}$ electron fluxes, measured at GEO by the GOES spacecraft, which are able to provide an accurate online forecast for 1 day ahead. One of the best online forecast models was implemented by the National Oceanic and Atmospheric Administration's Space Weather Prediction Center (NOAA-SWPC); however, even this forecast is very far from perfect. As such, one of the goals of this paper was to produce a model that would give a more accurate estimate than the model by NOAA-SWPC. This was achieved by validating the model on an interval of data, in other words, to see how the model forecasts would have performed during this interval.

\section{Methodology}

\subsection{NARMAX model}

The NARMAX approach, first developed by Leontaritis and Billings (1985a, b), is one of the most advanced data-based modelling techniques. It is a black box methodology that can automatically derive a model from solely input-output data. A multi-input single-output (MISO) NARMAX model was used to represent the dynamics of the electron fluxes at GEO. The general MISO NARMAX model can be represented by Eq. (1) (Billings et al., 1989; Billings and Tsang, 1989; Wei et al., 2004b):

$$
\begin{aligned}
y(t)= & F\left[y(t-1), \ldots, y\left(t-n_{y}\right),\right. \\
& u_{1}(t-1), \ldots, u_{1}\left(t-n_{u_{1}}\right), \ldots, \\
& u_{m}(t-1), \ldots, u_{m}\left(t-n_{u_{m}}\right), \ldots, \\
& \left.e(t-1), \ldots, e\left(t-n_{e}\right)\right]+e(t),
\end{aligned}
$$

where $y, u$ and $e$ are the output, input and noise respectively, $m$ is the number of inputs to the system and $n_{y}$, $n_{u_{1}}, \ldots, n_{u_{m}}$ are the maximum time lags of the output and the $m$ inputs respectively. $F[\cdot]$ is some nonlinear function and can be expanded in terms of polynomials, rational functions, B-Splines, radial basis functions etc. The noise terms 
of the NARMAX model allow the capture of noise entering the nonlinear system internally, resulting in coloured noise, rather than just an additive white noise (Billings et al., 1989; Chen et al., 1989; Billings and Chen, 1998)

There are three stages to identify a NARMAX model. The first stage, model structure detection, is to identify the most significant model terms by evaluating all the possible combinations of the past inputs, past outputs and past noise values with the use of the ERR. An advantage of the ERR in selecting input terms is that ERR is independent of the possible nonlinear and correlated noise (Billings, 2013). The second stage is parameter estimation, where the coefficients are calculated for each of the selected model terms in the structure detection stage. The inclusion of the noise terms can eliminate bias in estimating the coefficients. The final stage is model validation, using methods such as the correlation tests (Billings and Voon, 1986; Billings and Zhu, 1989) or model performance analysis. The full description of the NARMAX algorithm is very complex and, as such, it is beyond the scope of this paper. A complete description of the NARMAX algorithm can be found in the study of Billings et al. (1989).

The NARMAX algorithm requires both input and output data for the system to deduce a model. In this study, the output for each of the two models is the daily averaged $>0.8 \mathrm{MeV}$ electron flux and the $>2 \mathrm{MeV}$ electron flux. Both of these measurements are taken from the Geostationary Operational Environmental Satellite (GOES) at GEO and are supplied by the National Oceanic and Atmospheric Administration (NOAA) National Weather Service (NWS) Space Weather Prediction Center.

As discussed in the Introduction, previous data-based models have used geomagnetic indices and the solar wind velocity to forecast the electron fluxes at GEO. The recent study by Boynton et al. (2013) analysed the solar wind control parameters for a range of electron flux energies. They found that for energies $<1.8 \mathrm{MeV}$, the solar wind velocity was the most important solar wind parameter. However, the ERR results show that other solar wind parameters also play a minor role, such as density, and the $z$ component of the magnetic field in GSM coordinates. For higher energies ( $>1.8 \mathrm{MeV})$ the solar wind density is reported to have the most control of the electron fluxes but with the velocity still playing an important role. As such, the solar wind velocity $v$, density $n, z$ component of the IMF $B_{z}$ and Dst index were considered inputs for the models. Along with these parameters, the fraction of time that the solar wind remains southward within each day, $\tau_{B_{\mathrm{s}}}$, was also included. This parameter was calculated from the $1 \mathrm{~min} B_{z}$ by finding the time within each $24 \mathrm{~h}$ period that the IMF was southward and dividing it by the total time within the day. These data were from the Advanced Composition Explorer (ACE) spacecraft positioned at the L1 Lagrange and supplied by the OMNI website for training the model.

\subsection{Model training}

The NARMAX algorithm was then employed to obtain the two models for both the $>0.8 \mathrm{MeV}$ electron flux and the $>2 \mathrm{MeV}$ electron flux. This was achieved using sections of the data to train the models. These sections had to contain continuous equally sampled data, i.e. no data gaps within the interval for both input and output data.

The $>0.8 \mathrm{MeV}$ electron channel was used for the first time on the GOES 13, which only became operational on 14 April 2010. Therefore, there were not many data to train the model on, and the training data for the $>0.8 \mathrm{MeV}$ model were chosen to start on 10 April 2010 and end on 31 December 2010 .

The $>2 \mathrm{MeV}$ electron channel has been in use since GOES 6, and data are available from the late 1980s. Therefore, there were many more choices for the $>2 \mathrm{MeV}$ model training interval. This was chosen to start on 11 July 2004 and end on 11 October 2005.

The final models for both energies only included terms of past output, $v, n$ and $\tau_{B_{\mathrm{s}}}$, implying that both the Dst index and $B_{z}$ have a negligible influence on the electron fluxes. The models were then validated on separate validation data, which will be discussed in Sect. 3 .

\section{Model performance analysis}

Model performance analysis was used to validate the model and test whether the model would be accurate enough for real-time online forecasts of the 1-day-ahead electron flux at GEO. This was achieved using past data intervals to investigate how accurate the 1-day forecasts would have been compared to the electron flux observed by the GOES spacecraft.

Electron flux data from GOES 13 were used to evaluate the performance of the model. GOES 13 became the primary GOES satellite for the Energetic Proton Electron and Alpha Detector (EPEAD) on 14 April 2010. Thus, the period of data to analyse the $>2 \mathrm{MeV}$ electron flux model was from 14 April 2010 to 30 June 2012.

The previous GOES satellites measured the channel for $>2 \mathrm{MeV}$ electrons but not $>0.8 \mathrm{MeV}$ electrons; therefore, the $>0.8 \mathrm{MeV}$ electron channel was used for the first time on GOES 13. As mentioned above, the $>0.8 \mathrm{MeV}$ electron flux model was trained on data up to 31 December 2010, while the $>2 \mathrm{MeV}$ model was trained on data from 2005. Employing the data from 14 April 2010 to 31 December 2010 to evaluate the $>0.8 \mathrm{MeV}$ model would be bad practice. Therefore, the period of data to analyse the $>0.8 \mathrm{MeV}$ model could not be the same as the $>2 \mathrm{MeV}$ model and was instead from 1 January 2011 to 30 June 2012. Therefore, both models had different training and validation data.

The 1-day forecasts were calculated using the $>0.8$ and $>$ $2 \mathrm{MeV}$ models on the validation intervals mentioned above. Figure 1 depicts the measured (blue) and the model 1-day 


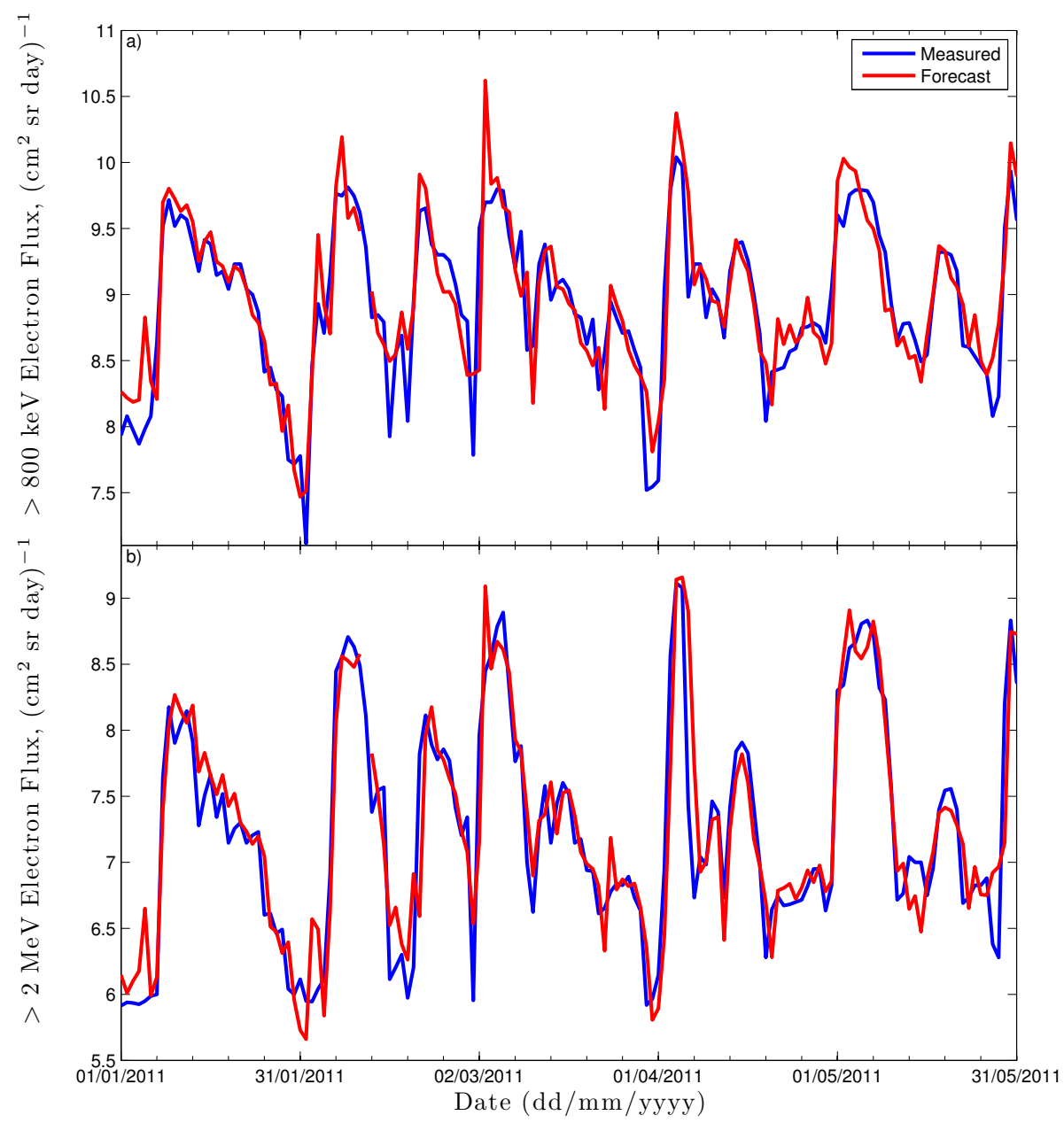

Figure 1. Model forecast showing measured electron flux in blue and the model estimate in red for (a) $>0.8 \mathrm{MeV}$ electron flux, (b) $>2 \mathrm{MeV}$ electron flux from 1 January 2011 to 31 May 2011.

forecast (red) for the period between 1 January 2011 and 31 May 2011. Figure 1a shows the $>0.8 \mathrm{MeV}$ model and Fig. $1 \mathrm{~b}$ the $>2 \mathrm{MeV}$ model. The $>2 \mathrm{MeV}$ model has a visibly better performance than the $>0.8 \mathrm{MeV}$ model; the $>0.8 \mathrm{MeV}$ model tends to overshoot when an increase in flux occurs. An issue with both these models (and other electron flux models) is that the model has a tendency to lag the measured flux. An example of this can be seen in Fig. 1 on 2 March 2011 for the $>0.8 \mathrm{MeV}$ model and 1 April 2011 for the $>2 \mathrm{MeV}$ model.

In addition to simply inspecting the figures showing the difference between the forecast and observed electron flux at GEO, the statistics of how the model 1-day forecast relates to the measurement needs to be calculated. Here, the performance of the models were statistically analysed using the prediction efficiency (PE) (Eq. 2), and the correlation coefficient (CC) (Eq. 3). These statistics are common in analysing the performance of models and have been used by Temerin and Li (2006), Li (2004), Boynton et al. (2011a) and Wei et al. (2004a) to name a few.

$$
\begin{gathered}
E_{\mathrm{PE}}=1-\frac{\sum_{t=1}^{N}\left[(y(t)-\hat{y}(t))^{2}\right]}{\sum_{t=1}^{N}\left[(y(t)-\bar{y}(t))^{2}\right]}, \\
\rho_{y \hat{y}}=\frac{\sum_{t=1}^{N}[(y(t)-\bar{y}(t))(\hat{y}(t)-\overline{\hat{y}}(t))]}{\sqrt{\sum_{t=1}^{N}\left[(y(t)-\bar{y}(t))^{2}\right] \sum_{t=1}^{N}\left[(\hat{y}(t)-\overline{\hat{y}}(t))^{2}\right]}},
\end{gathered}
$$

where $E_{\mathrm{PE}}$ is the PE, $\rho$ is the CC, $y(t)$ is the output at time $t, \hat{y}$ is the estimated output from the model and $N$ is the length of the data. The PE is a quantitative measure of the normalised error, where a high PE (unity) indicates a low error and a low PE $(\leq 0)$ shows that the error of the model is 
equivalent to (or greater than) the variance of the measured output and, thus, is very inaccurate. The CC is often employed as an indicator of a model's performance and shows the linear dependence between the measured and the forecast, unity showing high dependence and zero none.

The $>0.8 \mathrm{MeV}$ model resulted in a PE of 0.700 and CC of 0.847 between 1 January 2011 and 30 June 2012, while the $>2 \mathrm{MeV}$ model was found to have a PE of 0.786 and CC of 0.894 between 14 April 2010 and 30 June 2012. Therefore, both models show a high dependence between the measured and estimate, and an error much smaller than the measured electron flux variance. The lower PE for the $>0.8 \mathrm{MeV}$ model can be partly attributed to the overshoot observed in Fig. 1, which increases the error of the forecast.

\section{Comparison with NOAA-SWPC electron flux model}

The aim of this study was to derive electron flux models that provide a high accuracy for the 1-day forecast and implement them online in real time. The CC and the PE show that the models are accurate; however, since a high accuracy is relative, the $>2 \mathrm{MeV}$ electron flux model was compared to another online electron flux model. This model was the NOAASWPC model based on the work by Baker et al. (1990). This was the final hurdle that the model needed to pass before it was deemed suitable for real-time online implementation.

The SWPC provides a forecast of the $>2 \mathrm{MeV}$ electron flux at the website http://www.swpc.noaa.gov/products/ relativistic-electron-forecast-model. The forecast is calculated from the LPF model by Baker et al. (1990), which, as described in Sect. 1, employs the solar wind velocity, $\mathrm{Kp}$ index and AE index. The model has multiple modes: three that use ACE data, which estimate the 1-day-ahead, 2-day-ahead and 3-day-ahead electron flux at GOES. Only the 1-day-ahead estimate is considered here, since in this study the aim was to forecast 1 day in advance. The NOAA-SWPC provide statistics of the prediction efficiency for the previous year at http://services.swpc.noaa.gov/text/ relativistic-electron-fluence-statistics.txt. Between $29 \mathrm{Au}-$ gust 2011 and 28 August 2012 the PE of the SWPC model was $67.8 \%$. The prediction efficiency of the $\mathrm{SNB}^{3} \mathrm{GEO}>$ $2 \mathrm{MeV}$ electron flux model was calculated for the same time period and came to $77.5 \%$. Therefore, the NARMAX model had a $9.7 \%$ higher PE than the NOAA-SWPC for the same period of time.

\section{Conclusions}

The main aim of this study was to produce two electron flux models at GEO: for energies of $>0.8$ and $>2 \mathrm{MeV}$. Both of these models have been shown statistically to provide an accurate 1-day forecast for the validation period used in this study, as shown by the high prediction efficiencies and corre- lation coefficients between the 1-day forecasts and the measured fluxes.

The NARMAX $>2 \mathrm{MeV}$ model was compared to the NOAA-SWPC electron flux model (Baker et al., 1990). The PE for the NARMAX electron flux model was shown to be $\sim 10 \%$ higher than the SWPC model for the same period, illustrating that these models have the potential to provide an accurate real-time forecast for the following day's electron flux.

Thus, the goal to implement the models online to deliver a real-time forecast for the next day using the real-time data provided by the NOAA NWS Space Weather Prediction Center has been achieved and the online forecast of $\mathrm{SNB}^{3} \mathrm{GEO}$ electron flux models can be found at http://www.ssg.group. shef.ac.uk/USSW/UOSSW.html.

Acknowledgements. The authors would like to acknowledge the financial support from EPSRC and ERC. The authors would also like to thank the OMNIWeb service for providing the past solar wind data and the NOAA NWS Space Weather Prediction Center for the use of the real-time data from both ACE and GOES.

Topical Editor V. Fedun thanks two anonymous referees for their help in evaluating this paper.

\section{References}

Albert, J. M.: Evaluation of quasi-linear diffusion coefficients for emic waves in a multispecies plasma, J. Geophys. Res., 108, 1249, doi:10.1029/2002JA009792, 2003.

Albert, J. M.: Evaluation of quasi-linear diffusion coefficients for whistler mode waves in a plasma with arbitrary density ratio, J. Geophys. Res., 110, A03218, doi:10.1029/2004JA010844, 2005.

Baker, D. N., Higbie, P. R., Belian, R. D., and Hones, J. E. W.: Do jovian electrons influence the terrestrial outer radiation zone?, Geophys. Res. Lett., 6, 531-534, 1979.

Baker, D., Belian, R., Higbie, P., Klebesadel, R., and Blake, J.: Deep dielectric charging effects due to high-energy electrons in earth's outer magnetosphere, J. Electrostat., 20, 3-19, 1987.

Baker, D. N., McPherron, R. L., Cayton, T. E., and Klebesadel, R. W.: Linear prediction filter analysis of relativistic electron properties at 6.6 re, J. Geophys. Res., 95, 15133-15140, 1990.

Balikhin, M. A., Boaghe, O. M., Billings, S. A., and Alleyne, H. S. C. K.: Terrestrial magnetosphere as a nonlinear resonator, Geophys. Res. Lett., 28, 1123-1126, 2001.

Balikhin, M. A., Boynton, R. J., Walker, S. N., Borovsky, J. E., Billings, S. A., and Wei, H. L.: Using the narmax approach to model the evolution of energetic electrons fluxes at geostationary orbit, Geophys. Res. Lett., 38, L18105, doi:10.1029/2011GL048980, 2011.

Balikhin, M. A., Gedalin, M., Reeves, G. D., Boynton, R. J., and Billings, S. A.: Time scaling of the electron flux increase at geo: The local energy diffusion model vs. observations, J. Geophys. Res., 117, A10208, doi:10.1029/2011GL048980, 2012.

Billings, S. A.: Nonlinear System Identification: NARMAX Methods in the Time, Frequency, and Spatio-Temporal Domains, John Wiley \& Son Ltd, United Kingdom, 2013. 
Billings, S. A. and Chen, S.: The determination of multivariable nonlinear models for dynamic systems, in: Control and Dynamic Systems, edited by: Leondes, C. T., pp. 231-278, chapter 6, Academic Press, San Diego, 1998.

Billings, S. A. and Tsang, K. M.: Spectral analysis for nonlinear systems, part i: Parametric non-linear spectral analysis, Mech. Syst. Signal Proc., 3, 319-339, 1989.

Billings, S. A. and Voon, W. S. F.: Correlation based model validity tests for non-linear models, Int. Control J., 44, 235-244, 1986.

Billings, S. A. and Zhu, Q. M.: Nonlinear model validation using correlation tests, Int. Control J., 62, 749-766, 1989.

Billings, S., Chen, S., and Korenberg, M.: Identification of mimo non-linear systems using a forward-regression orthogonal estimator, Int. J. Control., 49, 2157-2189, 1989.

Blake, J. B., Kolasinski, W. A., Fillius, R. W., and Mullen, E. G.: Injection of electrons and protons with energies of tens of mev into $l<3$ on 24 March 1991, Geophys. Res. Lett., 19, 821-824, 1992.

Boaghe, O. M., Balikhin, M. A., Billings, S. A., and Alleyne, H.: Identification of nonlinear processes in the magnetospheric dynamics and forecasting of dst index, J. Geophys. Res., 106, 30047-30066, 2001.

Boscher, D., Bourdarie, S., Thorne, R., and Abel, B.: Influence of the wave characteristics on the electron radiation belt distribution, Adv. Space Res., 26, 163-166, 2000.

Boynton, R. J., Balikhin, M. A., Billings, S. A., Sharma, A. S., and Amariutei, O. A.: Data derived NARMAX Dst model, Ann. Geophys., 29, 965-971, doi:10.5194/angeo-29-965-2011, 2011a.

Boynton, R. J., Balikhin, M. A., Billings, S. A., Wei, H. L., and Ganushkina, N.: Using the narmax ols-err algorithm to obtain the most influential coupling functions that affect the evolution of the magnetosphere, J. Geophys. Res., 116, A05218, doi:10.1029/2010JA015505, 2011b.

Boynton, R. J., Balikhin, M. A., Billings, S. A., Reeves, G. D., Ganushkina, N., Gedalin, M., Amariutei, O. A., Borovsky, J. E., and Walker, S. N.: The analysis of electron fluxes at geosynchronous orbit employing a narmax approach, J. Geophys. Res.Space Physics, 118, 1500-1513, 2013.

Chen, S., Billings, S. A., and Luo, W.: Orthogonal least squares methods and their application to non-linear system identification, Int. J. Control., 50, 1873-1896, 1989.

Falthammar, C.-G.: Radial Diffusion by Violation of the Third Adiabatic Invariant, 157 pp., Reinhold, New York, 1968.

Freeman, J. W., O’Brien, T. P., Chan, A. A., and Wolf, R. A.: Energetic electrons at geostationary orbit during the November 3-4, 1993 storm: Spatial/temporal morphology, characterization by a power law spectrum and, representation by an artificial neural network, J. Geophys. Res., 103, 26251-26260, 1998.

Friedel, R., Reeves, G., and Obara, T.: Relativistic electron dynamics in the inner magnetosphere - a review, J. Atmos. Solar-Terr. Phys., 64, 265-282, 2002.

Fujimoto, M. and Nishida, A.: Energization and anisotropization of energetic electrons in the earth's radiation belt by the recirculation process, J. Geophys. Res., 95, 4265-4270, 1990.

Hilmer, R. V., Ginet, G. P., and Cayton, T. E.: Enhancement of equatorial energetic electron fluxes near $l=4.2$ as a result of high speed solar wind streams, J. Geophys. Res., 105, 23311-23322, 2000 .
Horne, R. B., Thorne, R. M., Glauert, S. A., Albert, J. M., Meredith, N. P., and Anderson, R. R.: Timescale for radiation belt electron acceleration by whistler mode chorus waves, J. Geophys. Res., 110, A03225, doi:10.1029/2004JA010811, 2005.

Ingraham, J. C., Cayton, T. E., Belian, R. D., Christensen, R. A., Friedel, R. H. W., Meier, M. M., Reeves, G. D., and Tuszewski, M.: Substorm injection of relativistic electrons to geosynchronous orbit during the great magnetic storm of March 24, 1991, J. Geophys. Res., 106, 25759-25776, 2001.

Kataoka, R. and Miyoshi, Y.: Flux enhancement of radiation belt electrons during geomagnetic storms driven by coronal mass ejections and corotating interaction regions, Space Weather, 4, S09004, doi:10.1029/2007JA012506, 2006.

Koons, H. C. and Gorney, D. J.: A neural network model of the relativistic electron flux at geosynchronous orbit, J. Geophys. Res., 96, 5549-5556, 1991.

Leontaritis, I. J. and Billings, S. A.: Input-output parametric models for non-linear systems part i: Deterministic non-linear systems, Int. J. Control., 41, 303-328, 1985a.

Leontaritis, I. J. and Billings, S. A.: Input-output parametric models for non-linear systems part ii: Stochastic nonlinear systems, Int. J. Control., 41, 329-344, 1985b.

$\mathrm{Li}, \mathrm{X}$.: Variations of $0.7-6.0 \mathrm{mev}$ electrons at geosynchronous orbit as a function of solar wind, Space Weather, 2, S03006, doi:10.1029/2003SW000017, 2004.

Li, X., Baker, D. N., Temerin, M., Larson, D., Lin, R. P., Reeves, G. D., Looper, M., Kanekal, S. G., and Mewaldt, R. A.: Are energetic electrons in the solar wind the source of the outer radiation belt?, Geophys. Res. Lett., 24, 923-926, 1997.

Ling, A. G., Ginet, G. P., Hilmer, R. V., and Perry, K. L.: A neural network-based geosynchronous relativistic electron flux forecasting model, Space Weather, 8, S09003, doi:10.1029/2010SW000576, 2010.

Liu, W. W., Rostoker, G., and Baker, D. N.: Internal acceleration of relativistic electrons by large-amplitude ulf pulsations, J. Geophys. Res., 104, 17391-17407, 1999.

McAdams, K. L., Reeves, G. D., Friedel, R. H. W., and Cayton, T. E.: Multisatellite comparisons of the radiation belt response to the geospace environment modeling (gem) magnetic storms, J. Geophys. Res., 106, 10869-10882, 2001.

O’Brien, T. P., Lorentzen, K. R., Mann, I. R., Meredith, N. P., Blake, J. B., Fennell, J. F., Looper, M. D., Milling, D. K., and Anderson, R. R.: Energization of relativistic electrons in the presence of ulf power and mev microbursts: Evidence for dual ulf and vlf acceleration, J. Geophys. Res., 108, 1329, doi:10.1029/2002JA009784, 2003.

Omura, Y., Furuya, N., and Summers, D.: Relativistic turning acceleration of resonant electrons by coherent whistler mode waves in a dipole magnetic field, J. Geophys. Res., 112, A06236, doi:10.1029/2010JA016280, 2007.

Reagan, J. B., Meyerott, R. E., Gaines, E. E., Nightingale, R. W., Filbert, P. C., and Imhof, W.: Space charging currents and their effects on spacecraft systems, Electrical Insulation, IEEE Transactions on, EI-18, 354-365, 1983.

Reeves, G. D.: Relativistic electrons and magnetic storms: 1992 1995, Geophys. Res. Lett., 25, 1817-1820, 1998.

Reeves, G. D., Chan, A., and Rodger, C.: New directions for radiation belt research, Space Weather, 7, S07004, doi:10.1029/2008SW000436, 2009. 
Schulz, M. and Lanzerotti, L. J.: Particle diffusion in the radiation belts, Phys. Chem. Space, Springer, Berlin, 81-113, 1974.

Sheldon, R. B., Spence, H. E., Sullivan, J. D., Fritz, T. A., and Chen, J.: The discovery of trapped energetic electrons in the outer cusp, Geophys. Res. Lett., 25, 1825-1828, 1998.

Shprits, Y. Y., Subbotin, D. A., Meredith, N. P., and Elkington, S. R.: Review of modeling of losses and sources of relativistic electrons in the outer radiation belt ii: Local acceleration and loss, J. Atmos. Solar-Terr. Phys., 70, 1694-1713, 2008.

Summers, D. and Thorne, R. M.: Relativistic electron pitchangle scattering by electromagnetic ion cyclotron waves during geomagnetic storms, J. Geophys. Res., 108, 1143, doi:10.1029/2002JA009489, 2003.

Summers, D., Thorne, R. M., and Xiao, F.: Relativistic theory of wave-particle resonant diffusion with application to electron acceleration in the magnetosphere, J. Geophys. Res., 103, 2048720500, 1998.

Summers, D., Ma, C., Meredith, N. P., Horne, R. B., Thorne, R. M., Heynderickx, D., and Anderson, R. R.: Model of the energization of outer-zone electrons by whistler-mode chorus during the October 9, 1990 geomagnetic storm, Geophys. Res. Lett., 29, 2174, doi:10.1029/2002GL016039, 2002.
Summers, D., Ma, C., Meredith, N., Horne, R., Thorne, R., and Anderson, R.: Modeling outer-zone relativistic electron response to whistler-mode chorus activity during substorms, J. Atmos. SolarTerr. Phys., 66, 133-146, 2004.

Temerin, M. and Li, X.: Dst model for 1995-2002, J. Geophys. Res., 111, A04221, doi:10.1029/2005JA011257, 2006.

Temerin, M., Roth, I., Hudson, M., and Wygant, J.: New paradigm for the transport and energization of radiation belt particles, Eos Trans. AGU, 75, 538, 1994.

Van Allen, J. A.: The geomagnetically trapped corpuscular radiation, J. Geophys. Res., 64, 1683-1689, 1959.

Wei, H. L., Billings, S. A., and Balikhin, M.: Prediction of the dst index using multiresolution wavelet models, J. Geophys. Res., 109, A07212, doi:10.1029/2003JA010332, 2004a.

Wei, H., Billings, S., and Lui, J.: Term and variable selection for nonlinear models, Int. Control J., 77, 86-110, 2004 b.

Wei, H.-L., Billings, S. A., Surjalal Sharma, A., Wing, S., Boynton, R. J., and Walker, S. N.: Forecasting relativistic electron flux using dynamic multiple regression models, Ann. Geophys., 29, 415-420, doi:10.5194/angeo-29-415-2011, 2011. 